\title{
ANALISIS DE LA IDENTIDAD PROFESIONAL A TRAVÉS DE LA COMPETENCIA EDUCADORA DE LA ENFERMERÍA
}

Chocarro González, Lourdes*, Guerrero Bonmatty, Rafael**, Venturini Medina, Carmen***, Salvadores Fuentes, Paloma****

*Prof. Titular Enfermería Medico Quirúrgica, **Enfermero. Licenciado en Antropología Social y Cultural. Prof. Titular Interino Enfermería Medico Quirúrgica, ***Enfermera. Prof. Titular Interina Enfermería Medico Quirúrgica, ****Enfermera. Licenciada en Sociología. Catedrática de Fundamentos de Enfermería.

Departamento de Enfermería. Facultad de Ciencias de la Salud. Universidad Rey Juan Carlos (URJC), Alcorcón Madrid

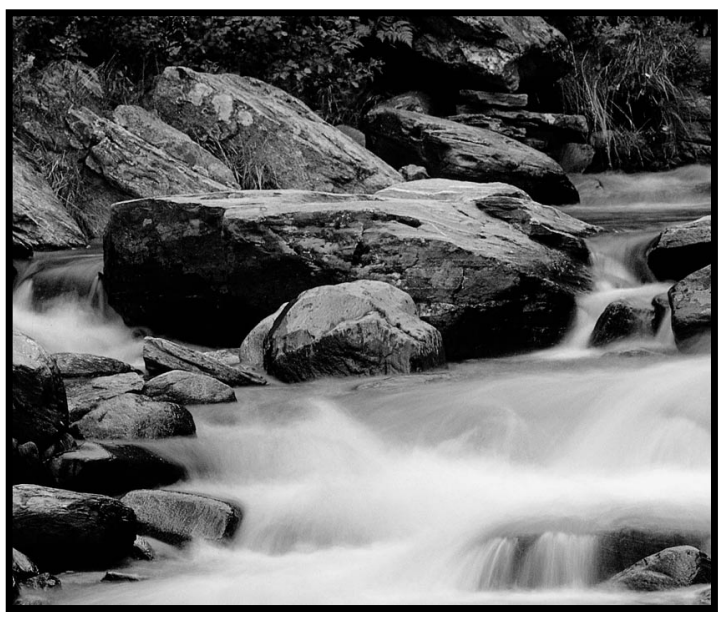

ANALYSIS OF PROFESSIONAL IDENTITY THROUGH NURSES' EDUCATIONAL COMPETENCE

\section{ABSTRACT}

The present article deals with nurses' perception of their own professional competence,

1 other health professionals' view and patients' social perspective.

Two discussion groups integrated by nurses working in two Madrid hospitals were constituted; most of our general study was carried out in these two hospitals. Both groups had different profiles, thus their discourses varied in many aspects, as can be seen in the analysis and conclusions.

The aim was to identify discourses elaborated by nurses interacting with other colleagues from the same hospital regarding educational competence towards hospitalised chronic patients.

From the analysis of discourses on the educational role, characteristics assigned to education by these two groups of nurses were identified, along with difficulties and conditioning factors.

The discussion groups own dynamics facilitated other discourses emerging in connection to the development of their educational role, such as social acknowledgement of the profession.

Key words: professional competence, educational activity, discussion groups, educational role, Nursing methodology, hospital.

\section{RESUMEN}

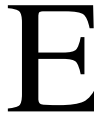

1 presente artículo se centra en la percepción que tienen las propias enfermeras sobre sus competencias profesionales, la visión que tienen otros profesionales sanitarios y la visión social que tienen los usuarios sobre este colectivo.

Para ello se configuraron dos grupos de discusión formados por enfermeras/os que trabajan en dos hospitales de Madrid, en cuyos ámbitos de trabajo se desarrolla gran parte de nuestro estudio general. Se seleccionaron dos grupos con perfiles diferentes, de manera que sus discursos en muchos aspectos, también son diferentes, como se podrá observar en el análisis y las conclusiones del trabajo.

Lo que se pretende es conocer los discursos que elaboraban las enfermeras/os, en interacción con 
otros compañeros de trabajo del mismo hospital, respecto de su competencia en educación con pacientes crónicos hospitalizados.

Analizando los discursos que fluían entorno al rol educativo, se pudo identificar las características que estos grupos de enfermeras/os asignaban a la educación que llevan a cabo en el hospital, así como la problemática derivada de su realización, y los factores que la condicionan.

La propia dinámica de los grupos de discusión, facilitó la emergencia de otros discursos que estas enfermeras/os ligaban de manera directa con el desempeño de su rol educativo, como son, el reconocimiento social de la profesión.

Palabras claves: competencias profesionales, actividad educadora, grupos de discusión, rol educativo, metodología de Enfermería, hospital.

\section{INTRODUCCIÓN}

El análisis del discurso en el seno de los grupos de discusión, es una herramienta útil que nos acerca al lenguaje de símbolos y representaciones sociales de las personas que configuran el grupo.

La sociología y la antropología hacen aportaciones interesantes respecto al análisis del discurso, como estrategia para acercarnos a las representaciones sociales, a las visiones del mundo. "Existe pues, una vinculación intrínseca entre discurso y poder, que se da tanto en el plano de las cosmovisiones como en el de la narrativa de identidad. Podemos, en consecuencia, analizar y evaluar en qué medida las formas discursivas contribuyen a sostener, reestructurar, desafiar o transformar relaciones de poder".

Analizando la problemática y la situación actual en la que se desarrolla la actividad educadora de la enfermera/o dentro del Hospital, nos acercamos a las competencias desempeñadas por las mismas en éste ámbito de actuación (Atención Especializada).

La construcción de la "Europa del conocimiento”, permitirá la formación de futuros profesionales capaces de desarrollar su trabajo en los distintos ámbitos que configuran el campo profesional europeo de Enfermería.

En España diferentes grupos están trabajando, tanto a nivel Comunitario, como Nacional para establecer, entre otras cosas, las competencias que deberán desempeñar los futuros Enfermeros en el marco de la Convergencia Europea.

En este artículo se presentan los resultados obtenidos en el marco del proyecto de investigación subvencionado por la CAM, en el año 2003 sobre la "Incidencia del rol educativo de enfermería en la frecuentación hospitalaria”, ya que el estudio de la actividad educadora de la Enfermería en el ámbito hospitalario, permite conocer todo el complicado entramado de aspectos que influyen en el desarrollo de esta actividad profesional.

¿Por qué un enfermero/a y no otro profesional? ¿Qué aportaciones específicas hace la enfermera/o en el ámbito del cuidado? ¿Cuál es su identidad profesional? ¿Cómo los ven sus compañeros? ¿Cómo los ven los usuarios?. En este sentido, se ha escrito mucho sobre la identidad profesional.

Es interesante conocer lo que dice el Diccionario de la Real Academia sobre la "competencia": "pericia, aptitud, idoneidad para hacer algo o intervenir en un asunto determinado".

En el presente estudio se pretende conocer las competencias en el área independiente de actuación en relación al desempeño del rol educativo con pacientes crónicos en Atención Especializada. Para ello hemos analizado los discursos elaborados por las enfermeras/os en interacción con otros compañeros que trabajan en el mismo hospital. Para tal fin se constituyeron dos grupos de discusión formados por enfermeras/os en activo, que tenían diferentes perfiles profesionales y desempeñaban su trabajo asistencial en dos hospitales públicos de la Comunidad de Madrid.

Analizando los discursos que fluyen en torno al rol educativo, se pudieron identificar las características que estos grupos de enfermeras/os asignaban a la educación que llevan a cabo en el hospital, así como la problemática derivada de su realización, y los factores que la condicionan, quedando de esta manera reflejado el modelo educacional directivo que se desempeña en los hospitales.

La propia dinámica de los grupos de discusión, facilitó la emergencia de otros discursos que estas enfermeras/os ligaban de manera directa con el desempeño de su rol educativo, como es, el reconocimiento social de la profesión: qué nivel de capacitación se otorgan a sí mismos, cuales son sus 
competencias y cuales sus funciones, qué imagen tienen tanto ellos mismos como la que tienen los usuarios del sistema público y la imagen que tienen los propios compañeros sanitarios, sobre el papel de Enfermería como profesión. Ello viene condicionado en gran medida por las competencias profesionales difusas y el reconocimiento social percibido por los profesionales.

Se detectan aspectos que tienen que ver con las características que conforman una profesión, entre las que destaca la identificación de un área independiente de actuación. En este sentido los grupos hablaban de la necesidad de aplicar una Metodología específica de Enfermería para garantizar los cuidados.

Los miembros de ambos grupos otorgan mucha importancia al desarrollo de la investigación en el área específica de Enfermería, que si bien se encuentran con dificultades para su desarrollo, lo identifican como un elemento clave que ayudará al impulso y consolidación de la profesión.

En el presente documento reflejamos textualmente aquellas expresiones más significativas en cada uno de los discursos que hemos identificado al respecto.

Por tanto podemos identificar los siguientes objetivos:

- Identificar las competencias que desarrolla el Diplomado de Enfermería en el área independiente de actuación respecto a las actividades educadoras.

- Describir la influencia que ejercen, la imagen y reconocimiento social en el desempeño de sus funciones.

- Describir el desempeño del rol educativo y el uso de la metodología de Enfermería.

\section{MATERIAL Y METODOS}

Se trata de un estudio descriptivo, en el que se ha aplicado metodología cualitativa, mediante la utilización de la estrategia del Grupo de Discusión.

El ámbito de estudio se circunscribe a dos Hospitales del Sistema Público de Salud, ubicados en la Comunidad de Madrid.

Se configuraron dos grupos de discusión, formados por enfermeras y enfermeros que trabajan en los mencionados ámbitos hospitalarios de la Comunidad de Madrid. Ambos grupos se configuraron con perfiles diferentes.
El desarrollo de los dos grupos de discusión tuvo lugar en el mes de Julio de 2002.

Los aspectos más destacables de los diferentes perfiles son los siguientes: uno de los grupos estaba compuesto por seis enfermeras, todas ellas son mujeres, de entre 30 y 45 años, con un amplio bagaje profesional (más de 7 años de desempeño de la profesión), que desempeñan su actividad profesional en un hospital de nivel 1 de la Comunidad de Madrid en Unidades de Hospitalización Médicas, Quirúrgicas y UCI de adultos. El otro grupo de discusión estaba compuesto por seis profesionales de Enfermería (un hombre y cinco mujeres), mucho más jóvenes que el anterior grupo, que han finalizado sus estudios recientemente (entre 2 y 7 años), y trabajan en un hospital de nivel 3 de la Comunidad de Madrid.

\section{COMPETENCIA EN EL DESEMPEÑO DEL ROL EDUCATIVO}

Las características que se destacan en relación a la educación que llevan a cabo las enfermeras/os con los pacientes hospitalizados crónicos son las siguientes:

- La importancia de utilizar un lenguaje asequible al paciente y que sea poco tecnificado.

- La educación está condicionada por el tiempo del que se dispone dentro de la jornada laboral es decir, la disponibilidad de tiempo limita su realización.

- Es necesario incluir al cuidador principal para llevar a cabo la educación a pacientes.

- Se necesita formación previa sobre cómo llevar a cabo la Educación en Salud.

El grupo de mayor experiencia profesional señala las siguientes dificultades de su puesta en marcha:

- El bajo nivel socioeconómico y cultural de los pacientes atendidos en este Hospital dificulta, en algunas ocasiones, la comprensión por parte de la población atendida respecto de la importancia que tiene en su proceso patológico cambiar hábitos no saludables

- Las enfermeras/os no detectan las necesidades de formación de los pacientes al ingreso

- Hay una falta de preparación y coordinación en el Alta del paciente entre las enfermeras/os y los médicos. 
- No disponen de recursos suficientes que garanticen un alta hospitalaria segura, es decir, que evite la aparición de complicaciones tras la llegada al domicilio del paciente.

- Parece existir escasa conexión del Hospital con Atención Primaria o con los Servicios Sociales.

El grupo de enfermeras/os con menor experiencia profesional destaca que:

- El estado de ánimo, el estrés, la presión asistencial, el interés de cada profesional son factores que influyen en la realización de la educación a los pacientes.

- La educación a pacientes está condicionada por las distintas formas de organización del trabajo.

- Falta formación para llevar a cabo una correcta Educación en Salud.

- Falta tiempo.

Los miembros de ambos grupos identifican como función propia el desempeño del rol educativo, sin embargo encuentran que su puesta en práctica es "decisión personal de cada enfermera/o". Este hecho es una deficiencia que imputan al profesional de Enfermería del hospital.

Se utiliza una metodología oral verbal. Educan poniendo ejemplos, dando nociones y comprueban el aprendizaje progresivo de habilidades técnicas mediante feed-back.

El modelo educativo que se sigue en ambos Centros no es participativo, sino direccional, en el que el protagonista es la enfermera/o. No se tiene en cuenta la necesidad de formación que manifieste o no el paciente, ni su nivel de información previo sobre el tema.

Informar al paciente es comunicarle aspectos que para la enfermera/o son importantes respecto de su proceso sin tener en cuenta ningún otro aspecto. Los objetivos de la educación parecen ser de la enfermera/o.

Las enfermeras/os manifiestan sentirse decepcionados cuando intentan modificar actitudes y no lo consiguen, sin haber tenido en cuenta las experiencias y visiones del propio paciente: "Tienes que aceptar que no vas a tener éxito y no vas a conseguir tus objetivos..." "Es frustrante todo lo que haces, tu trabajo no sirve para nada".
"Cuando das el Informe al Alta sabes que el paciente no va a hacer nada de lo que le has dicho".

Se apunta débilmente la importancia de la escucha activa, y la necesidad de mantener una actitud empática y de comprensión hacia el paciente en el momento de llevar a cabo la educación.

Destacamos algunas frases que hacen referencia al modelo educativo que parecen seguir estas enfermeras/os:

- "Los objetivos se bajan por comodidad". "El que falla no es el paciente, eres tú porque no transmites el mensaje adecuado.”.

- No se tiene en cuenta al paciente: "si el enfermo se niega a hacer algo que intentas educar, pues hay que empezar por otro lado." "No hay un patrón a seguir. Hay que averiguar por qué no quiere aprender."

- La educación se hace de forma general, " $a$ granel”. Se cuestiona la eficacia de la educación que da la enfermera porque "se trabaja poco en ella", deja mucho que desear en cuanto a las formas y canales de comunicación empleados.

- Se insiste en que la "educación es sistematizada y no individualizada: "a todo el mundo le das el mismo menú”. Dicen que la educación no es eficaz "no obedecen a la enfermera, lo sería si el paciente cambiara los hábitos."

- Asimismo se señala lo necesario que es otorgar al paciente un papel activo, pero no se ahonda en este aspecto. "El que sabe es el profesional".

Según Sánchez Moreno, estas afirmaciones corresponden a uno de los enfoques propuestos en su artículo, que tiene que ver con "La Educación para la salud informativa y prescriptiva" y que corresponde con un modelo autoritario que "obliga al paciente a seguir las normas”. La relación que se establece entre sanitario y paciente, en este modelo es vertical, propio de la medicina galénica, coherente con la elitización del conocimiento y la jerarquización social".

El grupo de mayor experiencia destaca el contexto social de dependencia médica y alta medicalización en que tradicionalmente se ha desarrollado la Enfermería, de manera que ha sido considerada como una "pseudoprofesión". De ahí la necesidad de desarrollar el área independiente de la profe- 


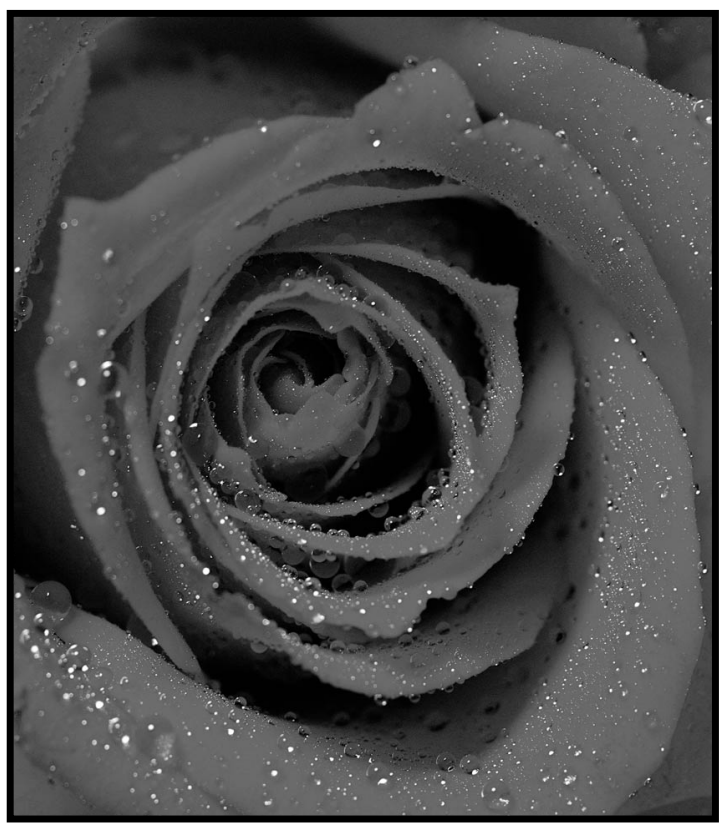

sión, mediante la utilización de un lenguaje propio (Diagnósticos de Enfermería) y un método de trabajo específicos (Planes de Cuidados).

Son conscientes de las dificultades que conlleva la utilización de estas herramientas "nuevas de trabajo" y se detecta un ambiente más propicio entre las enfermeras/os con más trayectoria profesional que en el grupo mas joven.

\section{IMAGEN PROPIA}

En este apartado se destacan las ideas que el colectivo tiene de sí mismo y la percepción del resto de profesionales sanitarios.

El reconocimiento social empieza por una visión nítida del las competencias que el colectivo tiene de su propia profesión, pero en el caso de las enfermeras/os están difusas: "la enfermera hace de todo". Estas se ven condicionadas por el resto de profesionales sanitarios que desarrollan su actividad, de manera muy estrecha con este colectivo, en el ámbito hospitalario. Médicos, Auxiliares y Celadores, valoran positivamente el trabajo de la enfermera/o, pero son incapaces de identificar la aportación específica que estos realizan.

Las enfermeras/os con mayor experiencia se encuentran altamente capacitadas, pero encuentran dificultades para poder llevar a cabo sus funciones. Estas dificultades provienen de la interacción con otros colectivos: "estamos demostrando que estamos en otro nivel, pero no nos dejan ocupar el puesto", "estamos preparadas, pero también nos pueden pedir que cojamos la fregona."

Dentro de las competencias identifican como suyo el rol educativo, pero opinan que "las competencias de Enfermería no están bien definidas: "Quizá ayudaría a esclarecer su aportación si las funciones estuviesen escritas: "Tenemos exceso de cargas de trabajo porque asumimos cosas que no son nuestras". "Registramos y escribimos como una autentica secretaria”. "La burocracia y los registros que cumplimentamos innecesariamente restan tiempo para educar. “

Se quejan de que la enfermera/o tiene integrado el rol asistencial por encima del rol educativo. La enfermera/o recibe una formación pre-grado centrada en el desarrollo de técnicas, situaciones patológicas, urgencias y se insiste poco en el desarrollo de otras competencias. Por ello no da importancia al rol educativo y consideran muy importante la labor asistencial. "La mayoría de las asignaturas eran de patologías”.

De forma unánime, opinan que todo el mundo, tanto las propias compañeras como el resto del personal, valoran más la actividad asistencial que cualquier otro tipo de actividad, de manera que " $s i$ no haces cosas con las manos no te consideran"; "si no te estas moviendo no estas haciendo nada útil"

En contraposición, se recoge que si se limitan a realizar las funciones más técnicas del profesional de Enfermería, podría llegarse a la sustitución de estos profesionales por otros de categoría inferior y formación profesional técnica "Si vas a limitar un montón de funciones terminará haciendo tu trabajo un técnico".

Existe un discurso entorno a la problemática del trabajo en equipo dentro del hospital, no sólo en el equipo de Enfermería formado por Enfermera y Auxiliar, sino también con el equipo Médico.

Identifican actividades independientes y dependientes, pero también hacen lo que el grupo denomina "trabajo subalterno", que consiste en hacer cosas que solo le sirven a otro profesional: "me traes, me sacas, me pones, me llevas", que nuevamente resta tiempo para desarrollar funciones independientes. 
Se ven a si mismos como un colectivo "conformista y muy crítico con sus pares. Se comunican poco entre ellas, se implican poco y no quieren adoptar más responsabilidades, de manera general."

Se reconocen como "la base dentro del equipo, como un elemento fundamental", pero la falta de reconocimiento a lo largo del desempeño de la profesión suele ser un factor de desmotivación para desarrollar más competencias.

Las funciones pueden estar identificadas según el modo de trabajo del hospital. La organización de cada equipo, parece que está condicionada por la “costumbre o la tradición”, pero no por una decisión tomada desde otro criterio más profesional.

Las enfermeras/os perciben que las Auxiliares valoran más positivamente a las enfermeras/os que les quitan trabajo: "cuantas mas cuñas pongas eres mejor enfermera/o”.

El médico considera que la buena enfermera/o es la que "es de plantilla, conocida y que resuelve".

Las enfermeras/os se consideran ubicadas entre las auxiliares y los médicos. Prima la actividad de colaboración con el médico y piensan que la enfermera/o no puede dejar de hacer determinadas cosas al paciente porque es insustituible, mientras que el médico sí puede dejar de hacerlo: "la tradición es ayudar al médico pero además debes prestar atención integral al paciente y no puedes dejar nada sin hacerle"

Un criterio que parece explicar el que algunos Equipos de Enfermería funcionen bien, parece ser "el llevarse bien y llevar mucho tiempo trabajando juntas"

Incluso aluden que dentro de la misma Unidad cada turno de trabajo, organiza el trabajo de manera diferente: "hay quien trabaja de la mano, otros van por separado", "toda la gente no tiene el mismo concepto de trabajo en equipo." "Dentro de un equipo hay distintas formas de ser y de actuar”.

Las enfermeras/os con mayor experiencia profesional se quejan por no conocer la actividad llevada a cabo en otras Unidades.

Piensan, en general, que no se trabaja en equipo y que el paciente lo percibe: "No hay Equipo de Enfermería". "Toda la gente no tiene el mismo concepto de trabajo en equipo." "Dentro de un equipo hay distintas formas de ser y de actuar”." Los pacientes dicen: hay formas de trabajo distintas... pero hacen su trabajo y me informan de los mismo”.

\section{IMAGEN SOCIAL QUE LAS ENFERMERAS CREEN QUE PERCIBEN LOS USUARIOS DEL SISTEMA}

Las enfermeras/os con mayor experiencia profesional piensan que los usuarios del Sistema Público de Salud, tienen una idea mas clara respecto de la contribución de las enfermeras que desempeñan su trabajo en los Centros de Atención Primaria de Salud: "alli si hay reconocimiento. La gente habla muy bien de ellas".

Al mismo tiempo y en contraposición, piensan que los pacientes que han pasado por el hospital, sobre todo crónicos, se sienten más satisfechos cuando están atendidos en el hospital, ya que les aporta mayor seguridad el trabajo de Enfermería Especializado. "luego no se fían de las enfermeras/os de Atención Primaria”, "el hospital les da seguridad".

Este grupo de profesionales opina que las enfermeras/os de Atención Primaria están poco formadas para mantener la continuidad de cuidados en algunos pacientes crónicos, y que estas instituciones (los Centros de Salud) cuentan con pocos medios, lo que dificulta aún más su actividad asistencial.

Faltaría conocer la opinión que tiene los usuarios, pero no se les ha incluído como informante en este artículo. Otros estudios realizados, dicen que los usuarios discrepan respecto a la opinión de los expertos, y ven alas enfermeras/os desde una imagen "anclada en ideas de tipo religioso, maternal, y con carácter de dependencia; no hay noción de la complejidad del rol profesionalizante".

\section{ROL EDUCATIVO Y METODOLOGÍA DE ENFERMERÍA}

Ambos grupos identifican la utilidad del uso de los Diagnósticos de Enfermería (DdE) ya que contribuye a identificar el área específica e independiente de actuación de la enfermera/o, pero son muchas y variadas las dificultades que se encuentran tanto en su uso como en las estrategias de 
implantación. Se identifican los diagnósticos enfermeros como parte importante de la autonomía de la profesión.

De los dos grupos de discusión emergen las dificultades para la puesta en marcha de metodología de cuidados que incluya el uso de los Diagnósticos de Enfermería (DdE).

No se identifica un claro rechazo hacia los DdE pero sí hay cierto recelo. Se reconoce que la Dirección de Enfermería otorga importancia al tema, aunque las estrategias de implantación no están libres de fallos, "el marco teórico va por un lado y no tiene nada que ver con la realidad".

Ambos grupos identifican la importancia de contar con unos buenos registros, ya que favorecen el desarrollo de la profesión.

Muchos registros de los que se cumplimentan deriva de la burocracia de otros profesionales, generalmente del médico, y restan tiempo para dedicar al paciente y a los registros de Enfermería.

Entre las dificultades expresadas por ambos grupos en relación al registro de estas actividades destacamos los siguientes:

- "Que no hay costumbre de registrar". "No se organiza la planificación y por tanto no queda por escrito".

- Requieren tiempo para su cumplimentación. "Se necesita mas tiempo para registrar. Es importante registrar porque lo que no está registrado no está hecho". "Registramos y escribimos como una autentica secretaria”, "Tenemos exceso de cargas de trabajo porque asumimos cosas que no son nuestras".

El Informe al Alta de Enfermería pretende ser el registro indicador específico de la planificación y continuidad de los cuidados. En él debe quedar reflejada la actividad enfermera, siendo parte de ésta, la educación llevada a cabo con el paciente.

Este Informe se realiza acorde a criterios que debe cumplir el paciente. El grado de cumplimentación de éste no es el mismo en todas y cada una de las Unidades de Hospitalización de adultos: "Se hace con determinados enfermos y dependiendo del Servicio donde se genere el Alta".

La falta de institucionalidad en la cumplimentación del Informe se ve reflejada en el contenido de los mismos: "no están adecuados a la metodología de enfermería”.

\section{CONCLUSIONES}

Existe coincidencia tanto en el grupo de menor como en el de mayor experiencia en que la actividad educativa se lleva a cabo en el hospital, y se reconoce como una función propia.

El medio social hospitalario no ayuda en la puesta en práctica de la Educación en Salud. Los turnos, la presión asistencial, la falta de formación al respecto, son algunos de los aspectos que frenan el desarrollo de una actividad educadora rica y dinámica.

Los pacientes crónicos demandan información a las enfermeras/os.

Entre el propio colectivo todavía se sigue valorando mas la actividad asistencial que cualquier otro tipo de actividades, al que restan importancia.

Las enfermeras/os identifican los factores socioeconómicos y culturales de sus pacientes como elementos a tener en cuenta a la hora de garantizar un Alta segura al domicilio, y no encuentran demasiados recursos para evitar peligros a sus pacientes.

El modelo educativo que se sigue es directivo. Posiblemente esté condicionado por el ambiente y limitaciones que impone la dinámica cotidiana de la vida de las enfermeras en el hospital.

Se sienten reconocidas pero algo infravaloradas en el desempeño de su trabajo, tanto por los usuarios como por los otros compañeros sanitarios.

Las enfermeras/os se sienten altamente capacitadas para el desempeño de multitud de funciones, y al mismo tiempo se sienten muy responsables de sus pacientes.

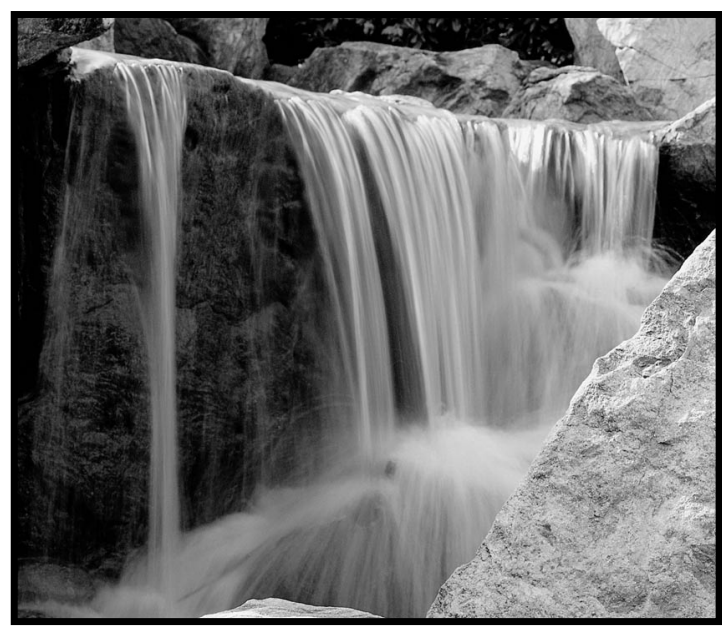


Cada equipo de Enfermería se organiza el trabajo de maneras muy diferentes y los criterios son variados.

Los grupos identifican el uso de un lenguaje específico de Enfermería (DdE) y de una metodología propia como la clave para la consolidación del colectivo como profesión.

Se deben analizar los recursos humanos y técnicos con los que se cuentan para adaptar las metas a conseguir.

Hay que destacar, como necesidad prioritaria, cambiar la metodología de trabajo y pasar del modelo biomédico a un modelo centrado en los Cuidados de Enfermería.

Los registros son reconocidos por las enfermeras/os como instrumentos indispensables para documentar y evaluar su actuación. Actualmente hay un reto importante al respecto para documentar la calidad de los cuidados y las acciones cuidadoras de las enfermeras/os.

Los registros transmiten la información necesaria para la atención de un enfermo entre distintos profesionales sanitarios, pero están poco o nada desarrollados los aspectos más específicos relacionados con la transmisión de información entre los propios profesionales de Enfermería, utilizando un lenguaje propio.

En el análisis del discurso de estos grupos, se vislumbra que las enfermeras/os identifican mejor los problemas interdependientes que los problemas independientes.

La actividad enfermera se ve determinada por la motivación y compromiso individual. Influyen el bajo reconocimiento social y la "desmotivación a lo largo del desempeño de la profesión por falta de nuevas metas de futuro".

La actividad técnica - asistencial prima por encima de cualquier otra actividad de carácter más intelectual. Esta actividad técnica proporciona mayor seguridad y mayor reconocimiento al profesional. Se evita la implicación en actividades que requieren mayor compromiso. Las enfermeras/os que participan en grupos de trabajo suelen hacerlo fuera del trabajo asistencial cotidiano. Asimismo, se identifica como gran condicionante para el desarrollo de actividades que no son puramente asistenciales, tener que mantener las presencias físicas, así como la organización de las plantas de hospitalización, y el problema que suscita la ausencia de una enfermera en la Unidad durante su jornada laboral.

Ambos grupos coinciden en que el desarrollo de la investigación en el ámbito de la enfermería mejoraría su imagen social, aumentaría la motivación profesional y se fomentaría el desarrollo de la profesión en múltiples y variados ámbitos.

\section{BIBLIOGRAFÍA}

- ALBERDI, R. M. (1992). "La identidad profesional de la enfermera". Revista Rol de Enfermería n ${ }^{\circ} 170$, 39-45.

- ARIÑO, A. (1997). Ideologías, discursos y dominación. Revista Española de Investigación (REIS). 79, 179-220.

- Diccionario de la lengua española (2001). Vigésima segunda edición.

- GARCÍA, A y col. (2000). Imagen social de la enfermera. Desarrollo Científico Enfermería. Volumen 8 $\mathrm{n}^{\circ} 9$, octubre

- SALVADORES, P, y col. (2003). Incidencia del rol educativo de Enfermería en la frecuentación hospitalaria. Número de proyecto Ref 08.7/0004/2001.1.año 2003

- SALVADORES, P, y col. (2003). Red de Enfermería para la Convergencia con Europa. Comunidad Autónoma de Madrid- ORDEN 6533/2002, de 26 de noviembre, del Consejo de Educación.

- SÁNCHEZ, A. (1995). Estructura de racionalidad en educación para la salud: fundamentos y sistematización. Rev sanitaria Hig Pub. 69; 1, 5-15.

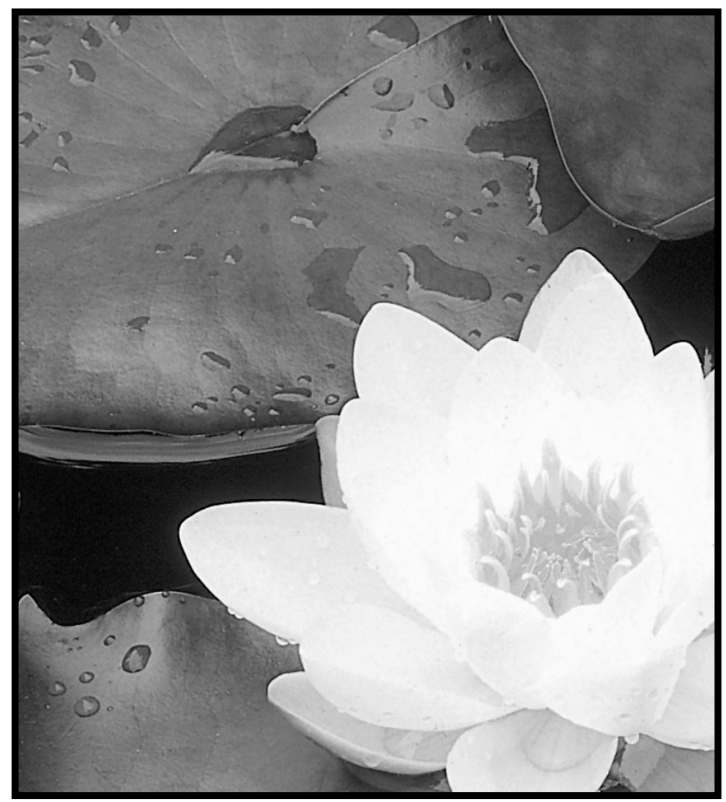

